\title{
Neutrophils Are Decreased in Obsessive-Compulsive Disorder: Preliminary Investigation
}

\author{
Murad Atmaca $^{1}{ }^{凶}$, Faruk Kilic ${ }^{1}$, Filiz Koseoglu' ${ }^{1}$ and Bilal Ustundag ${ }^{2}$ \\ ${ }^{1}$ Departments of Psychiatry and ${ }^{2}$ Clinical Biochemistry, FIrat University, School of Medicine, Elazig, Turkey
}

\begin{abstract}
Objective There has been no study in the literature evaluating total blood count in obsessive-compulsive disorder (OCD). Therefore, we performed the present study to spesifically measure serum total blood count particularly white blood cells to see whether or not its eventual alterations might have an etiopathogenetic significance in patients with OCD.

Methods Total blood count was measured in thirty patients and same number of healthy controls. Additionally, all patients were assessed by Yale-Brown Obsession Compulsion Scale (Y-BOCS).

Results Except for neutrophil count, there were no significant differences between the groups regarding any haematological parameter. The mean neutrophil count of the patient group was lower compared to that of the control subjects.

Conclusion In conclusion, the present study suggests that neutrophil count is reduced in pure OCD patients and this finding may contribute to the role of immunological factors in the pathogenesis of OCD.

Psychiatry Investig 2011;8:362-365
\end{abstract}

Key Words OCD, Total blood count, Neutrophil.

\section{INTRODUCTION}

Obsessive-compulsive disorder (OCD) comprising core symptoms of obsessions and compulsions is a common, chronic, anxiety disorder that can have disabling effects throughout lifetime with a wide range of clinical pictures. Its etiopathogenesis is obscure despite a huge different data coming from genetic, neurobiological, neurochemical and neuroimaging investigations. The contemporary knowledge indicates that OCD has a multifactorial etiopathogenesis. Recently, there has been an important advance in the neurobiology of OCD. It has been established that severe brain traumas, impairments in biochemical neurotransmission and receptor functions may be associated with the occurrence of OCD. Meanwhile, growing evidence implicates that the immunologic factors may be associated with the pathophysiology of some subtypes of OCD, called as Paediatric Autoimmune

Received: March 18, 2011 Revised: June 15, 2011

Accepted: June 15, 2011 Available online: November 3, 2011

$\triangle$ Correspondence: Murad Atmaca, MD

Department of Psychiatry, Firat (Euphrates) Üniversitesi, Firat Tip Merkezi Psikiyatri Anabilim Dali, 23119 Elazig, Turkey

Tel: +90-424-233-3555/2282, Fax: +90-424-238-7688

E-mail: matmaca_p@yahoo.com

(a) This is an Open Access article distributed under the terms of the Creative Commons Attribution Non-Commercial License (http://creativecommons.org/licenses/bync/3.0) which permits unrestricted non-commercial use, distribution, and reproduction in any medium, provided the original work is properly cited.
Neuropsychiatric disorders Associated with Streptococcal infections. ${ }^{1}$ In fact, the relationship between infectious disease and OCD has extended to the year of 1918. In that year, it was observed a great influenza pandemy in which the patients who had recovered from von Economo's encephalitis began to show a variety of psychiatric and neurologic sequelae. Denys et al. ${ }^{2}$ measured ex vivo production of tumor necrotising factor (TNF- $\alpha$ ), interleukin-4 (IL-4), IL-6, IL-10, and interferon (IFN- $\gamma$ ) in whole blood cultures, and natural killer (NK) cell activity and peripheral blood NK cell-, monocytes, T-cell-, and B-cell-percentages were measured in 50 medication-free outpatients with OCD and 25 controls and found a significant decrease in production of TNF- $\alpha$ and NKactivity compared to healthy controls, without any significant differences in the other immune variables. Therefore, they commented that changes in TNF- $\alpha$ and NK activity might be a potential role of altered immune function in the pathophysiology of OCD. Moreover, beyond these limited investigations, to date, no study has evealuated total blood particularly white blood cells' (WBC) count in the patients with OCD. When we take a look the other psychiatric disorders, it can be observed a dearth of investigations on total blood count. In their study, Fan et al. ${ }^{3}$ evaluated outpatients who were 19 to 75 years old diagnosed with schizophrenia or schizoaffective disorder participated in a multi-center, cross- 
sectional study by measuring their levels of glucose, lipids and $\mathrm{WBC}$ and demonstrated that $\mathrm{WBC}$ strongly predicted the condition of metabolic syndrome after controlling some factors such as age, gender, race, age of illness onset, family history of diabetes, and smoking status and found significant correlations between WBC and Brief Psychiatric Symptom Scale. total score, negative symptom score and anxious depression factor scores.

To the best of our knowledge, there has been no study in the literature evaluating total blood count in OCD. Therefore, we performed the present study to spesifically measure serum total blood count particularly WBC to see whether or not its eventual alterations might have an etiopathogenetic significance in patients with OCD.

\section{METHODS}

Thirty patients with pure OCD (23 females and 7 males; mean age $=31.83 \pm 5.49$ years) according to DSM-IV from the Firat University Scool of Medicine Department of Psychiatry who were met the admission criteria were enrolled in the present study. After providing written informed consent, each subject underwent a physical examination and a psychiatric diagnostic evaluation. A DSM-IV diagnosis of OCD was established on the basis of independent clinical interviews by one senior psychiatrist. All subjects gave informed written consent after the process of the study was fully explained. The study was performed in accordance with the Declaration of Helsinki.

Exclusion criteria included serious and unstable medical illnesses, existence of any Axis I comorbidity, the history of any endocrinological condition, females who are pregnant or lactating, or of childbearing potential, substance dependence within the previous 3 months, known medical conditions which might affect changes in metabolic parameters, known history of diabetes or lipid disorder, use of anti-diabetic or lipid-lowering therapy or special diets to affect haematological parameters, use of anti-inflammatory agents or clinically relevant abnormal laboratory tests including megaloblastic anemia.

Avaliable 30 healthy control subjects ( 23 females and 7 males; mean age $=31.17 \pm 4.62$ years; range $21-45)$ according to exclusion criteria were chosen from the hospital staff. They had not current or lifetime history of any psychiatric disorders. They did not also have any history of major mood disorder, dementia, and psychosis in their first degree relatives. In addition, they did not have the history of any endocrinological condition, lactating, or of childbearing potential, substance dependence within the previous 3 months, known medical conditions which might affect changes in metabolic parameters, known history of diabetes or lipid disorder, use of anti-diabetic or lipid-lowering therapy or special diets to affect haematological parameters, use of anti-inflammatory agents or clinically relevant abnormal laboratory tests including megaloblastic anemia. Controls were matched with the patients in regard to sex and age.

The patients were administered Yale-Brown Obsession Compulsion Scale (Y-BOCS) ${ }^{4}$ to determine the severity of OCD. Smoking was prohibited after $23.00 \mathrm{~h}$, one day before blood drawing.

\section{Blood sampling and determination of folate and homocysteine}

Venous blood samples from left forearm vein were collected into $5 \mathrm{~mL}$ vacutainer tubes containing potassium EDTA between 7 and 8 a.m after overnight fasting. Hematological parameters were measured by using an autoanalyzer (Coulter Max M, Coulter Electronics Ltd, Luton, UK).

\section{Statistical analysis}

The data were analyzed using SPSS (version 13.0; SPSS Inc., Chicago, IL). Demographics, clinical measures and laboratory values were reported using descriptive statistics. Group comparisons were carried out by using independent samples $t$ test. The chi-square test was used to compare the categorical variables. For correlation evaluations, the Spearman correlation (two-tailed) test was used. $\mathrm{p}<0.05$ was considered to be significant.

\section{RESULTS}

There were no significant differences in age, socioeconomic status, living place and female/male ratio between the patients and control groups $(\mathrm{p}>0.05)$. In regard to smoking status which was accepted as $>20$ cigarettes per day, no difference was found between groups, with eleven in the patient group versus nine in control ones ( $p>0.05)$. Sociodemographic and clinical variables are presented in Table 1 . The mean Y-BOCS scores were $27.50 \pm 5.09$ and $8.20 \pm 2.12$ for the patients and controls, respectively $(\mathrm{p}<0.001)$.

The results of the measurements of the hematological parameters in the patient groups and controls are summarized in Table 1.

Except for neutrophil count, there were no significant differences between the groups regarding any haematological parameter. The mean neutrophil count of the patient group was lower compared to that of the control subjects (3.89 \pm $1.19 / \mathrm{mm}^{3}$ versus $\left.4.69 \pm 1.72 / \mathrm{mm}^{3} ; \mathrm{p}<0.01\right)$. Six of the patients (24\%) from the patient group and two (8\%) from controls had low neutrophil count when taking into consideration 
Table 1. Demographic and hematological parameters

\begin{tabular}{lcc}
\hline & $\begin{array}{c}\text { OCD patients } \\
(\mathrm{N}=25)\end{array}$ & $\begin{array}{c}\text { Controls } \\
(\mathrm{N}=25)\end{array}$ \\
\hline Age & $31.83 \pm 5.49$ & $31.17 \pm 4.62$ \\
Gender (F/M) & $23 / 7$ & $23 / 7$ \\
Y-BOCS & $27.50 \pm 5.09$ & $8.20 \pm 2.12^{*}$ \\
WBC & $7.20 \pm 1.68$ & $7.92 \pm 2.09$ \\
Neutrophil & $3.89 \pm 1.19$ & $4.69 \pm 1.72^{* *}$ \\
Lymphocyte & $2.63 \pm 0.95$ & $2.26 \pm 0.99$ \\
Platelet & $287.57 \pm 54.79$ & $277.17 \pm 83.90$ \\
Red blood cells & $4.82 \pm 0.42$ & $4.88 \pm 0.54$ \\
Hemoglobine & $14.15 \pm 1.51$ & $13.73 \pm 1.51$ \\
Hematocrit & $41.68 \pm 4.18$ & $40.35 \pm 4.25$ \\
\hline
\end{tabular}

${ }^{*} \mathrm{p}<0.001,{ }^{* *} \mathrm{p}<0.05$. OCD: obsessive compulsive disorder, F/M: female/male, Y-BOCS: Yale-Brown Obsession Compulsion Scale, WBC: white blood cell

normal neutrophil levels $(\mathrm{p}<0.05)$. In regard to smoking, when comparing the smokers to the non-smokers both in the patient and control groups, no differences were determined except for red blood cells (RBC). The mean RBC count was higher in smokers than in nonsmokers both in OCD patients $\left(6.34 / \mathrm{mm}^{3}\right.$ versus $4.12 / \mathrm{mm}^{3}$; in smokers and non-smoker, respectively; $\mathrm{p}<0.01)$ and in the controls $(6.34 /$ $\mathrm{mm}^{3}$ versus $4.12 / \mathrm{mm}^{3}$; in smokers and non-smoker, respectively; $\mathrm{p}<0.01)$. There were no effects of gender on the hematological parameters of patient and control groups ( $\mathrm{p}>0.05)$.

There were no any correlations between Y-BOCS scores and any haematological parameters including neutrophil counts both in the patient and control groups ( $p>0.05)$. In addition, neutrophil count was not correlated to the duration of illness, and age ( $\mathrm{p}>0.05)$.

\section{DISCUSSION}

The present study demonstrated that the mean neutrophil count of the patient group was lower compared to that of the control subjects and six of the patients (24\%) from the patient group and two (8\%) from controls had low neutrophil count when taking into consideration normal neutrophil levels $(p<0.05)$. First of all, we should emphasize that this is the single study evaluating complete blood count in OCD. In their study, Denys et al. ${ }^{2}$ found that OCD patients had a significant decrease in TNF- $\alpha$ and NK activity, relative to controls whereas they had not altered other cytokines (IL-4, IL10 , IF- $\gamma$ ) and immune cells (granulocytes, monocytes, B-lymphocytes, T-lymphocytes, and NK-cells). In this discussion, we should focus on our main finding, decreased neutrophil count. A growing evidence revealed neutrophil to be on the one hand a target and on the other hand a source of proinflammatory cytokines such as IL- 1 and TNF- $\alpha$, chemokines like IL-8, and growth factors including granulocyte/ monocyte colony stimulating factor. On the other hand, it has been reported that these proinflammatory cytokines particularly TNF- $\alpha$ and chemokines may also function as priming agents of neutrophils, notably increasing their phagocytosis, degranulation, and oxidative responses. ${ }^{5}$ There has been interactional relationship between TNF- $\alpha$ and central neurotransmitter activity particularly serotonin. Mössner et al. ${ }^{6}$ reported that serotonin transporter function might be increased by TNF- $\alpha$. Both Denys et al. ${ }^{2}$ and Konuk et al. ${ }^{7}$ reported altered TNF- $\alpha$ values in OCD compared to healthy ones. This alteration seems to be associated with decreased neutrophil count because of the fact that neutrophils are both a target and a source of proinflammatory cytokines including TNF- $\alpha$. Moving these discussions, of course, just a decreased neutrophil count may account fort he whole pathogenesis of OCD, however, we can speculate that because there is an important communication between the immune system and central nervous system altered neutrophil count and consequently immune function in OCD would reflect a change in neurotransmission which is an important role of its pathophysiology.

The findings found in the present study should be evaluated with some limitations. First, because the sample size was relatively small, we could not generalize the findings to the all OCD patients. However, at this point we should emphasize the strength of the study which included OCD patients without any comorbidity. Second, we only examined complete blood count, related parameters could be evaluated.

In conclusion, the present study suggests that neutrophil count is reduced in pure OCD patients and this finding may contribute to the role of immunological factors in the pathogenesis of OCD.

\section{REFERENCES}

1. Swedo SE, Leonard HL, Garvey M, Mittleman B, Allen AJ, Perlmutter $\mathrm{S}$, et al. Pediatric autoimmune neuropsychiatric disorders associated with streptococcal infections: clinical description of the first 50 cases. Am J Psychiatry 1998;155:264-271.

2. Denys D, Fluitman S, Kavelaars A, Heijnen C, Westenberg H. Decreased TNF-alpha and NK activity in obsessive-compulsive disorder. Psychoneuroendocrinology 2004;29:945-952.

3. Fan X, Liu EY, Freudenreich O, Park JH, Liu D, Wang J, et al. Higher white blood cell counts are associated with an increased risk for metabolic syndrome and more severe psychopathology in non-diabetic patients with schizophrenia. Schizophr Res 2010;118:211-217.

4. Goodman WK, Price LH, Rasmussen SA. The Yale-Brown Obsessive Compulsive Scale. Arch Gen Psychiatr 1989;46:1006-1016.

5. Witko-Sarsat V, Halbwachs-Mecarelli L, Schuster A, Nusbaum P, Ueki I, Canteloup S, et al. Proteinase 3, a potent secretagogue in airways, is present in cystic fibrosis sputum. Am J Respir Cell Mol Biol 1999;20: 729-736.

6. Mössner R, Heils A, Stöber G, Okladnova O, Daniel S, Lesch KP. En- 
hancement of serotonin transporter function by tumor necrosis factor alpha but not by interleukin-6. Neurochem Int 1998;33:251-254.

7. Konuk N, Tekin IO, Ozturk U, Atik L, Atasoy N, Bektas S, et al. Plasma levels of tumor necrosis factor-alpha and interleukin-6 in obsessive compulsive disorder. Mediators Inflamm 2007;65704. 